\title{
A Modified Fast Marching Method for Defining Fiber Placement Trajectories Over Meshes
}

\author{
M Bruyneel $^{1}$ and S. Zein ${ }^{1}$
}

\begin{abstract}
In this paper, we study the efficiency of applying the numerical simulation of a moving interface to the problem of defining fiber placement trajectories for composite structures. This new approach, which is based on an adaptation of the Fast Marching method, presents two advantages. Firstly, it simulates accurately the mechanism of an Automated Fiber Placement (AFP) machine by producing equidistant fiber courses, which avoids avoiding undesirable gaps or overlaps between the successive courses, while allowing to exploit the full capacity of the machine. Secondly, it is able to handle complex surfaces by working directly on the mesh instead of the geometric equation. Consequently, this new approach directly provides the local orientation of the fibers inside each mesh element, what is relevant for a finite element structural analysis. This approach is illustrated through some numerical examples taken from real case studies, for straight and curved fiber trajectories.
\end{abstract}

Keywords: structural composite(A), modeling(C), fiber placement.

\section{Introduction}

In the Automated Fiber Placement (AFP) or Automated Tow Placement (ATP) technologies (see [1]), the head of the machine lays down multiple

\footnotetext{
${ }^{1}$ LMS Samtech division, Liege Science Park, rue des chasseurs ardennais 8 B4031 Angleur Belgium, Michael.bruyneel@samtech.com, corresponding autor.
} 
pre-impregnated tows on the structure. As reported in [2], AFP machines can place simultaneously up to 32 tows of $1 / 8$ in. side by side during a course of the machine head. A complete ply is obtained by placing courses side by side. An advantage of AFP with regards to the Automated Tape Laying (ATL) process is that it allows the definition of curved fiber paths using narrow individual tows. The AFP process can therefore handle structures of complicated shape with possibly non straight fiber courses. A review of the different ways to place the fiber courses is provided in [2].

Even if the draping simulation of fabrics has been extensively studied in [3-6]. Only a small amount of research has been devoted to the computer definition of the fiber trajectories in AFP. Hyer and Gürdal, in [7-10], were amongst the first authors to propose a computer base solution for variable stiffness laminates in flat panels.

In $[4,5]$, the case of the woven fabrics is considered. The weft and wrap directions are defined by computing the crossing points of the fibers. These points constitute a grid of points with a fixed spacing in each direction and belong to the draping surface. The fibers are the broken lines connecting the crossing points.

Another approach for defining curved fibers is having a general function for the fiber orientation over the draping surface. In [11], the fibers are placed on a flat surface and assumed to be a set of Bezier curves or polynomial functions. In $[2,10,12]$, the fibers are defined as a geodesic path, a constant angle path, a path with a linearly varying fiber angle, or a path with constant curvature. They have been applied on cones and cylinders.

A third approach in [13-15] is to consider a reference curve over the draping surface and to compute the set of points over the surface which are at a constant distance from the reference curve. A fiber is the broken line that 
connects these equidistant points.

All of these approaches suffer from two major drawbacks. First, they are geometry methods requiring the parametric equations of the draping surfaces. In most industrial cases, these parametric equations are not available. Usually, the draping surface is defined using a CAD software that generates a mesh of it. Therefore, one needs a method for defining the curved fibers using a mesh and not parametric equations.

Secondly, these approaches (except the third one) do not generate equidistant paths for the courses in the AFP process. If two consecutive course centerlines are not equidistant, undesired gaps and overlaps apprear (see Figure 1). The gaps weaken the structure and the overlaps increase its weight. In [2] it is mentioned that using the cut and restart capabilities of the AFP machine allows to avoid the undesired gaps and the overlaps, and consequently provides plies of constant thickness over the structure. However, this technique can be time expensive since the full capability of the machine to propose courses of constant (and maximum) width is not used.

In this paper, it is proposed to adapt a numerical method, called the Fast Marching Method (FMM), to define equidistant courses over surfaces of general (and possibly complex) geometry. The Fast Marching Method is used in many fields of the physics for the simulation of a moving interface [16]. The approach developed here relies on the definition on the structure of a reference curve, which represents the initial position of the interface, and which can be seen as an initial fiber trajectory represented by a course centerline. This reference curve is called "reference fiber" from now on. This reference fiber is then propagated over the whole surface in such a way that (possibly) curved fibers trajectories are generated without the drawbacks of gaps and overlaps, while exploiting the full capacity of the AFP machine, i.e. laying down courses of 
constant (and maximum) width. Moreover, this approach works with a mesh of the surface rather than using parametric equations to define the geometry of the structure. This feature makes possible the definition of fiber trajectories for structures of complex geometry. Finally, since the method provides the local fiber orientation in each element of a mesh, it is a natural input for the finite element method [17] when a structural analysis of the composite component must be conducted.

The paper is organized as follows. In Section 2, the general mathematical formulation for the simulation of a moving interface and the methodology proposed in this paper are presented. In Section 3, the algorithm developed for the definition of the reference fiber is explained. Sections 4 and 5 concentrate on the propagation of this initial information over the whole surface using the standard FMM method. In Section 6, several adaptations are brought to the FMM in order to handle the specific problem of defining suitable equidistant fiber trajectories. In Section 7, the local fiber orientation is obtained in each element of the mesh representing the surface. Finally, a set of numerical experiments are presented in Section 8 to show the efficiency of the new approach. It covers the cases of planar and non planar complex structures with straight and curved reference fibers.

\section{The Mathematical Formulation}

The definition of the set of equidistant fibers is based on the solution of the Eikonal equation. It is a non-linear partial differential equation which has many applications in the domain of interface propagation. This equation predicts the travel time of a moving interface over a domain.

Let $\Omega$ be a bounded computational domain in $\mathbf{R}^{3}$ representing the shell structure to be draped and $\Gamma$ be a reference curve defined on the structure 

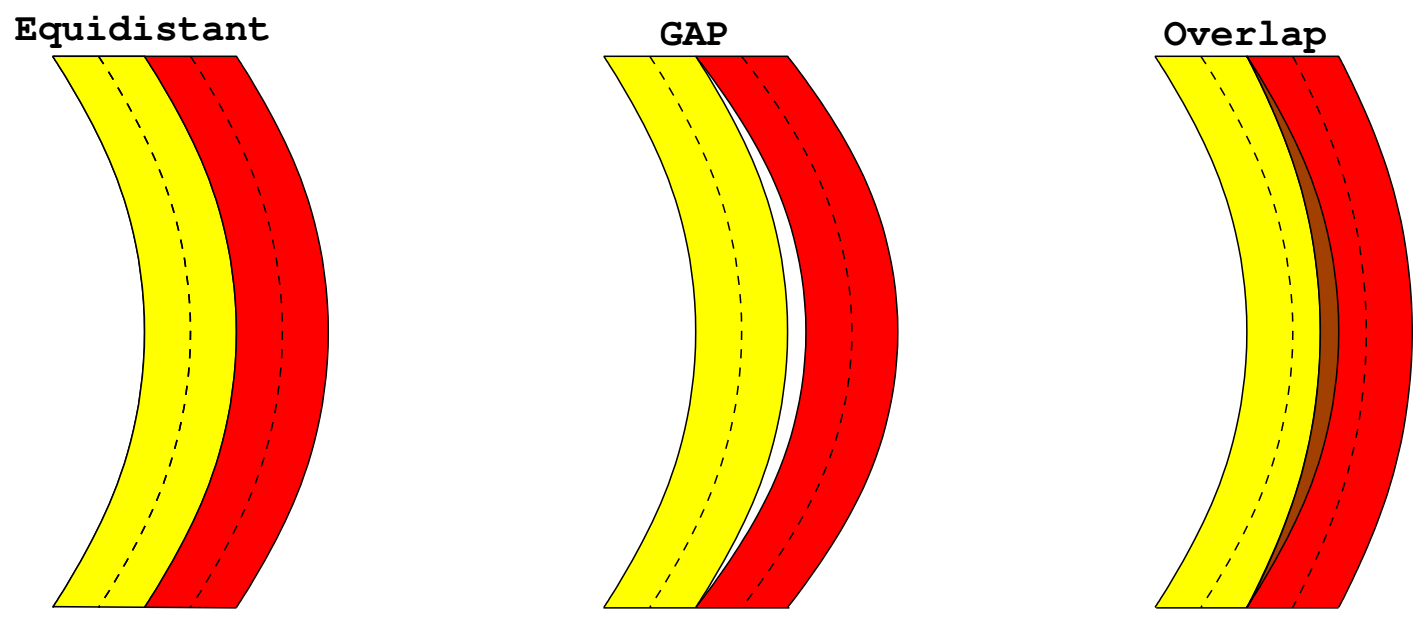

Figure 1: Gaps and overlaps between two consecutive fiber courses due to non-equidistant fibers.

representing the reference fiber. The set of equidistant fibers generated from $\Gamma$ is defined as follows. The reference fiber $\Gamma$ is propagated over the whole domain $\Omega$. Each point of the curve has a constant speed orthogonal to the curve. Let $T(x)$ be the travel time needed by $\Gamma$ to travel to point $x$ at a speed equal to $1 / f . T(x)$ is also proportional to the distance from $x$ to $\Gamma$. One can directly see that any equidistant curve to $\Gamma$ is an iso-value of $T$ (see Figure 2). This constitutes the basis of our work.

The travel time $T(x)$ is given by the Eikonal equation 1 :

$$
\left\{\begin{array}{l}
|\nabla T(x)|=f, x \in \Omega \backslash \Gamma \\
T(x)=0, x \in \Gamma \subset \Omega
\end{array}\right.
$$

It is supposed that this equation is solved numerically over a triangular mesh representing the structure and $T$ is computed at the nodes of the mesh.

Quadrangular mesh elements can be managed since they are transformed into triangles.

Generating the set of equidistant fibers is a process of three steps:

1. tracing the reference curve $\Gamma$ (the reference fiber) over the mesh to define 


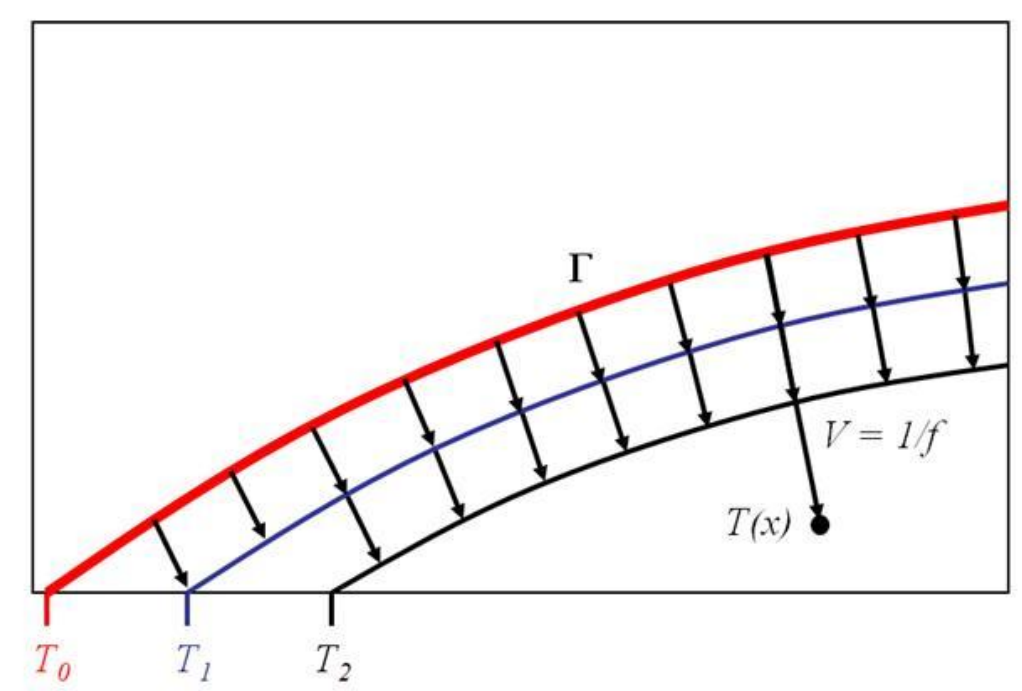

Figure 2: Propagation of the initial curve and equidistant curves at different instants.

the initial condition of equation (1),

2. solving numerically equation (1) to compute $T$ at the nodes of the mesh,

3. computing from $T$ the orientation of the iso-value (the fibers) at each cell of the mesh.

Figures 3-6 illustrate the principle of the method on a flat surface, in a rectangular domain, knowing that the approach can be applied to non developpable surfaces if they are represented by a mesh. These steps are detailed in the following sections.

\section{The Tracing of the Reference Fiber}

The reference fiber $\Gamma=\Gamma(t)$ is a $3 \mathrm{D}$ curve that belongs to the surface representing the structure $(\Omega)$. The tangent to $\Gamma(t)$ can be decomposed into two components, $\dot{\Gamma}_{\perp}(t)$ and $\dot{\Gamma}_{\|}(t)$, which are respectively orthogonal and parallel to $\Omega$. Since each point of $\Gamma(t)$ belongs to $\Omega$, it can be shown that $\dot{\Gamma}_{\perp}(t)=0$ and $\dot{\Gamma}_{\|}(t)=\dot{x}(t)$, where $x(t)$ is a given 2D function. This function represents the shape of the reference fiber. The curvilinear abscissa $t$ is in an 


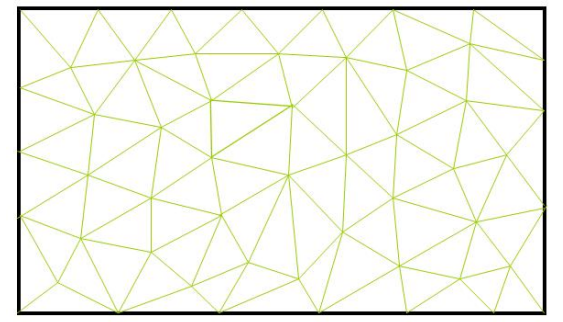

Figure 3: Define the mesh of the structure.

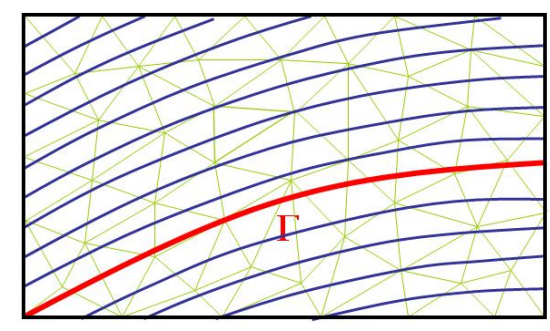

Figure 5: Solve the Eikonal equation.

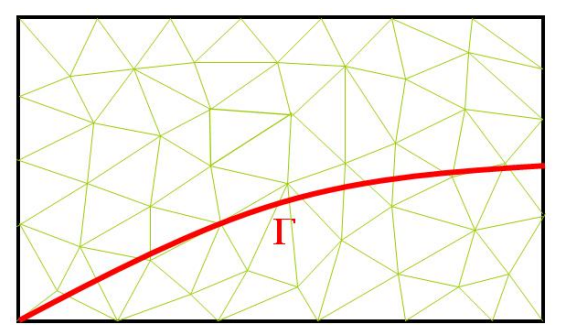

Figure 4: Trace the reference fiber.

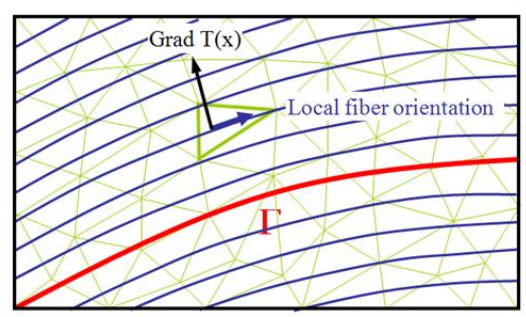

Figure 6: Compute the fiber orientation for each element.

interval $\left[0, t_{\max }\right]$ with $t_{\max }$ sufficiently large such that the two points $\Gamma(0)$ and $\Gamma\left(t_{\max }\right)$ belong the to boundary of $\Omega$.

The structure over which the reference fiber is traced is discretized with a triangular mesh $\Omega_{h}$. The tracing algorithm consists in finding the intersection points of $\Gamma(t)$ with the edges of the elements of the mesh $\Omega_{h}$. The reference curve $\Gamma$ intersects each mesh element with two points: one entry point and one exit point. For each mesh element and given the entry point, the tracing algorithm finds the exit point and the next element into which the reference curve enters.

At the initialization step (see Figure 7), $x(t)$ has to be defined in the $x O y$ plane independently from the mesh. $x(t)$ can be a straight line, a cubic spline or any other 2D parametric curve. It is supposed that $x(0)$ is at the origin of the axes $x O y$. $\Gamma(0)$, the starting point of $\Gamma$ over the mesh, is defined by giving: the edge $P Q$ to which it belongs, its corresponding element $P Q R$ and the ratio $\lambda_{1}$ by which $\Gamma(0)$ divides the edge $P Q$. Two points $A$ and $B$ are defined on the 


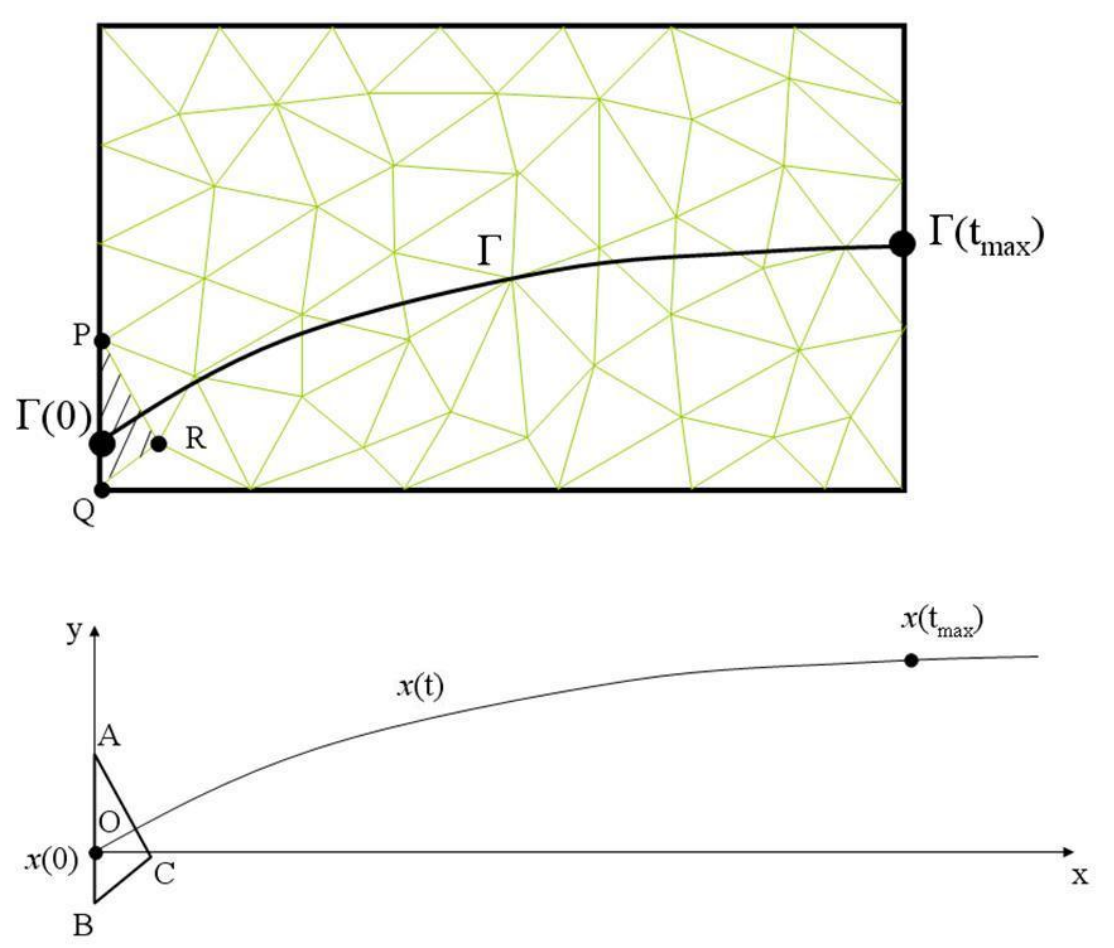

Figure 7: Tracing the reference fiber over the mesh from $x(t)$.

y-axis such that $\overrightarrow{A O}=\lambda_{1} \overrightarrow{A B}$.

Take an index $k=0$ and $t_{k}=0$. At each iteration $k$, construct in the plane $x O y$ a triangle $A B C$ identical to the mesh element $P Q R$ such that $x(t)$ enters $A B C$ for $t \geq t_{k}$. Find the edge $I J$ that $x(t)$ intersects. $I J$ can be $A B, A C$ or $B C$. Then, find the intersection abscissa $t_{k+1}$ and the intersection point $H=x\left(t_{k+1}\right)$. Compute $\lambda_{2}$, the ratio by which $H$ divides the edge $I J$. Let $U V$ be the corresponding edge of $I J$ in the mesh $P Q R$. One can deduce that $\Gamma$ intersects $U V$ at point $G$ such that $\overrightarrow{U G}=\lambda_{2} \overrightarrow{U V}$. Find the other element that has $U V$ in common with $P Q R$. Pose $P=U, Q=V, A=I, B=J, \lambda_{1}=\lambda_{2}$ and $k=k+1$.If this other mesh does not exist, then pose $t_{\max }=t_{k+1}$ and stop. Otherwise, perform a new iteration.

$\Gamma(t)$ is approximated by $\Gamma_{h}(t)$, the broken line that connects the intersection points. 


\section{The Local Solver of the Eikonal Equation}

The full algorithm is described in details in $[16,18]$. Since it is well documented in the literature, it is reproduced here only for the case of an acute triangular element in the mesh, for the sake of clarity of the paper. For more details on this local solver and the case of an obtuse triangle, the reader should refer to[16, 18]. The problem to solve here can be stated as follows: knowing the travel times at two nodes of a mesh element, how can we propagate this information to the third node of the element.

Consider $A B C$ an acute mesh element, as illustrated in Figure 8. The local solver is an algorithm that updates the travel time $T_{C}$ at vertex $C$ given the travel times $T_{A}$ and $T_{B}$. It locally simulates the propagation of the fiber inside the element $A B C$ using a first order approximation. The angles of the vertices $A, B$ and $C$ are respectively $\alpha, \beta$ and $\gamma$. The fiber is locally linear inside the mesh element $A B C$ and this segment has to pass by $C$ (Huygen's principle). As explained in $[16,18]$, this condition is satisfied if and only if (see Figure 8):

- $\left(T_{B}-T_{A}\right) / f \leq A B$ : the fiber can pass by $A$ then $B$ in a time $\left(T_{B}-T_{A}\right)$ given its speed $1 / f$,

- $\theta \leq \beta$ : the fiber passes by $B$ before $C$,

- $\theta+\beta \leq \pi / 2$ : the orthogonal projection of $\mathrm{C}$ on the fiber falls inside $A B C$ which means that $C$ is updated from $A$ and $B$.

These considerations lead to the following local solver in algorithm 1. Note that the min is used because the update of $C$ using the local solver can be repeated several times according to the Fast Marching Method presented in the next section. Therefore, the smallest value of $T_{C}$ must be kept. For more details on this local solver and the case of $A B C$ obtuse see $[17,18]$ 


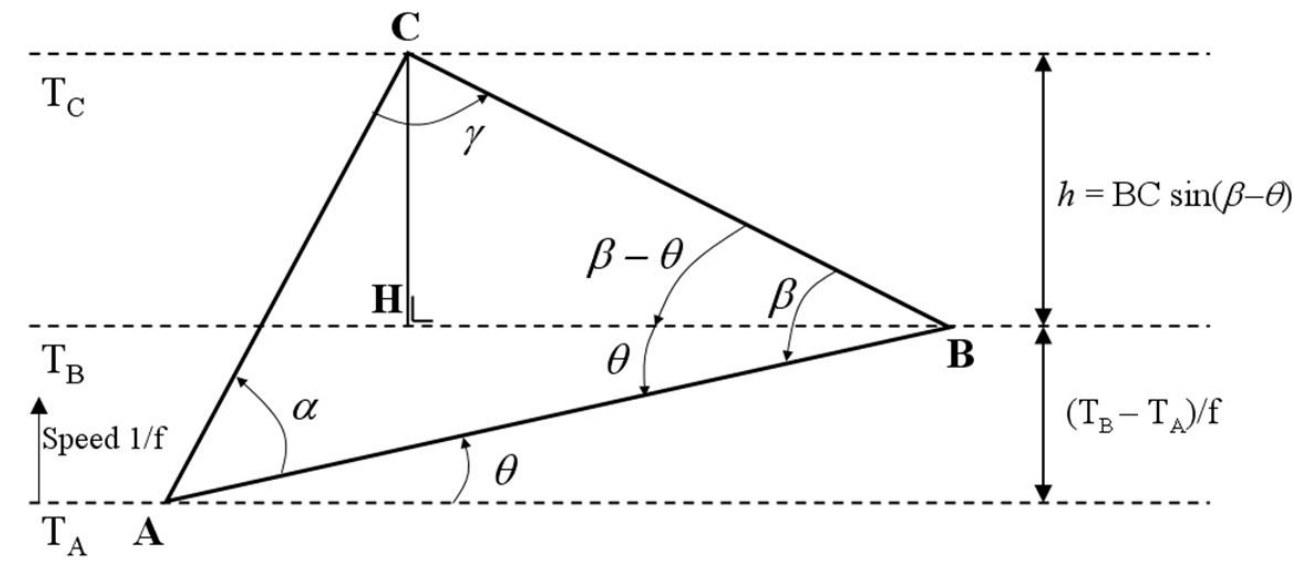

Figure 8: Updating $T_{C}$ from $T_{A}$ and $T_{B}$.

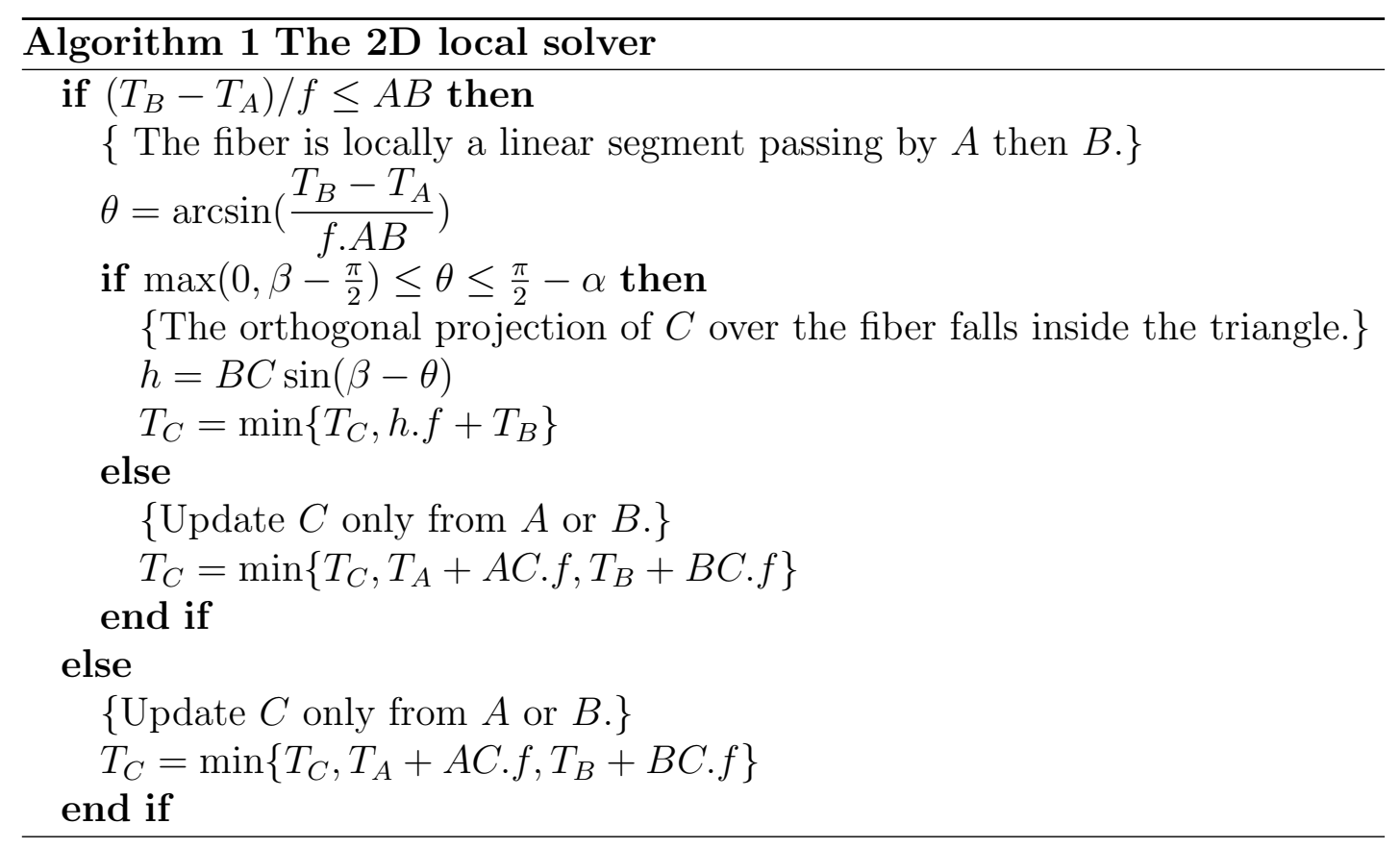




\section{The Fast Marching Method}

In this section, the Fast Marching Method (FMM) is recalled. This method is used for tracking the motion of an interface animated with a speed $1 / f$, always normal to itself. It aims to simulate the complete propagation of the reference fiber by tracking its motion inside each element using the local solver of the previous section. The algorithm computes at each node $x$ the instant $T(x)$ at which the interface has passed. After computing theses instants at all the nodes, the position of the interface at an instant $T$ is the iso-value curve $T(x)=T$.

At the initialization step of the algorithm and after tracing the reference fiber over the mesh, the nodes of the mesh are gathered into three groups: frozen, close and far. The frozen nodes are the set of the nodes of the elements through which the reference fiber has passed. The close nodes are the set of nodes which are neighbors of the frozen nodes. The far nodes are the remaining nodes (see Figure 9 ). The $T(x)$ of each frozen node is initialized to its distance to the reference fiber times $f$. The $T(x)$ of the other nodes are initialized to infinity. Finally, for each node $C$ in the close set and for each mesh element $A B C, T(C)$ is updated from $T(A)$ and $T(B)$ using the local solver described above.

The FMM relies on the principle that the information propagates from the smaller values of $T$ to the larger ones. This is known as the upwind scheme. The moving interface is in the region between the frozen and the close nodes.

At each iteration, let $C$ be the node in the close set which has the smallest value of $T$. Remove this node of the close set and add it to the frozen set. Let $M$ be the set of neighbors of $C$ in the far set. Move the nodes of $M$ from the far to the close set. For each element $A B M$ update $T_{M}$ from $T_{A}$ and $T_{B}$ using 


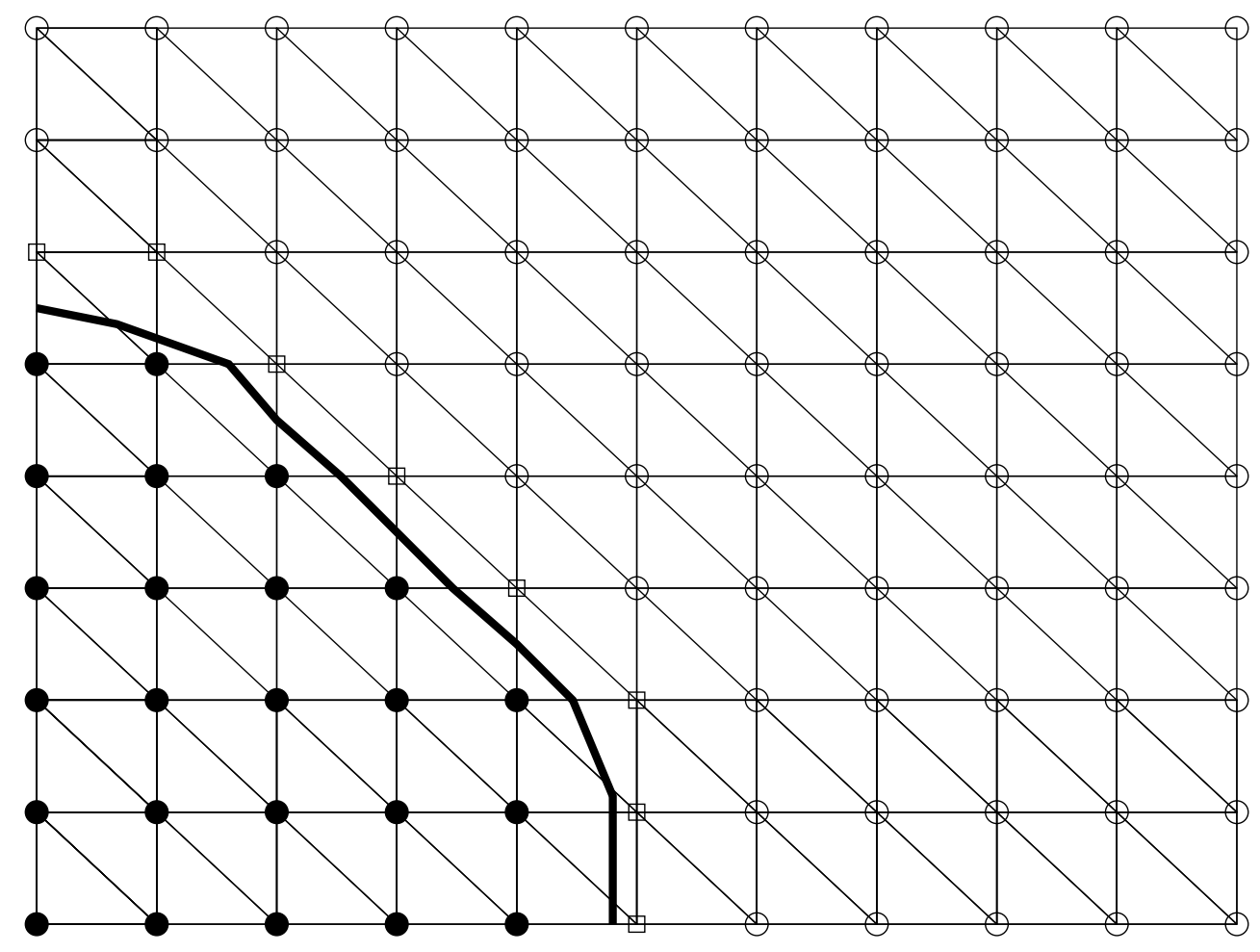

Figure 9: The fast marching method: the froze nodes (black dots), the close nodes (white squares) and the far nodes (white dots).

the local solver. The algorithm must terminate when all the nodes of the domain are in the frozen set.

For a mesh made of $\mathrm{N}$ nodes, the FMM has a total operation count of $\mathrm{O}(\mathrm{N} \log \mathrm{N})$ to find the $\mathrm{T}(\mathrm{x})$ values over the whole meshed structure. For more details on the fast marching method, refer to $[17,18]$.

\section{Adaptation of the Method}

In the two situations illustrated in Figures 10 and 11, the fast marching method does not exactly produce the expected network of fibers, even if the solution consists in equidistant trajectories. Consider the first case in Figure 10 where straight equidistant fibers are to be defined over a rectangular domain. The reference fiber is a bounded segment. This segment propagates in the band defined by the two orthogonal lines at the two ends of the segment. The region 
outside this band is filled with arcs of circles. Even if the FMM provides a set of equidistant trajectories, the final network of fibers is not the expected one, this last being illustrated in Figure 12. The solution to this problem is to simulate the propagation of an infinite reference fiber rather than a bounded one. This means that the nodes at the boundary of the domain are updated from the part of the fiber which is outside the domain, given that the reference fiber is infinite (see Figures 13 and 14). The 2D local solver is then modified as follows. An additional condition is added to $\max \left(0, \beta-\frac{\pi}{2}\right) \leq \theta \leq \frac{\pi}{2}-\alpha$. Now, the point $C$ is updated using its projection $H$ over the fiber in two cases: if $H$ is inside the triangle $A B C$ (the preceding condition) or if $C$ belongs to the boundary of the domain and $H$ is outside the domain (see figures 13 and 14).

The second case which requires an adaptation of the algorithm is the one of a concave domain where the fiber reaches the critical point where the boundary is tangent to the fiber. Indeed, even with the adaptation of the algorithm proposed in Figure 13, a correct solution for the problem of Figure 11 is not obtained, as reported in Figure 15. To simulate the infinite fiber, one needs to extend it like in Figure 16. In the 2D local solver, if the point $C$ belongs to the boundary and the tangent to the boundary at $C$ is parallel to the fiber, the fiber is extended using the tracing algorithm described in section 3.

\section{Computing the fiber orientations in each element of the mesh}

For each element $M N P$ of the mesh and from the computed values $T_{M}, T_{N}$ and $T_{P}$, one has to compute the angle between the fiber passing though $M N P$ and the edge $M N$. It is assumed that $T$ inside $M N P$ is a linear function and the fiber is an iso-value of $T$.

Firstly, construct in the plane $x O y$ a triangle $A B C$ identical to $M N P$. Secondly, find the linear function of the form $\tilde{T}=a \cdot x+b \cdot y+c$ that interpolates 


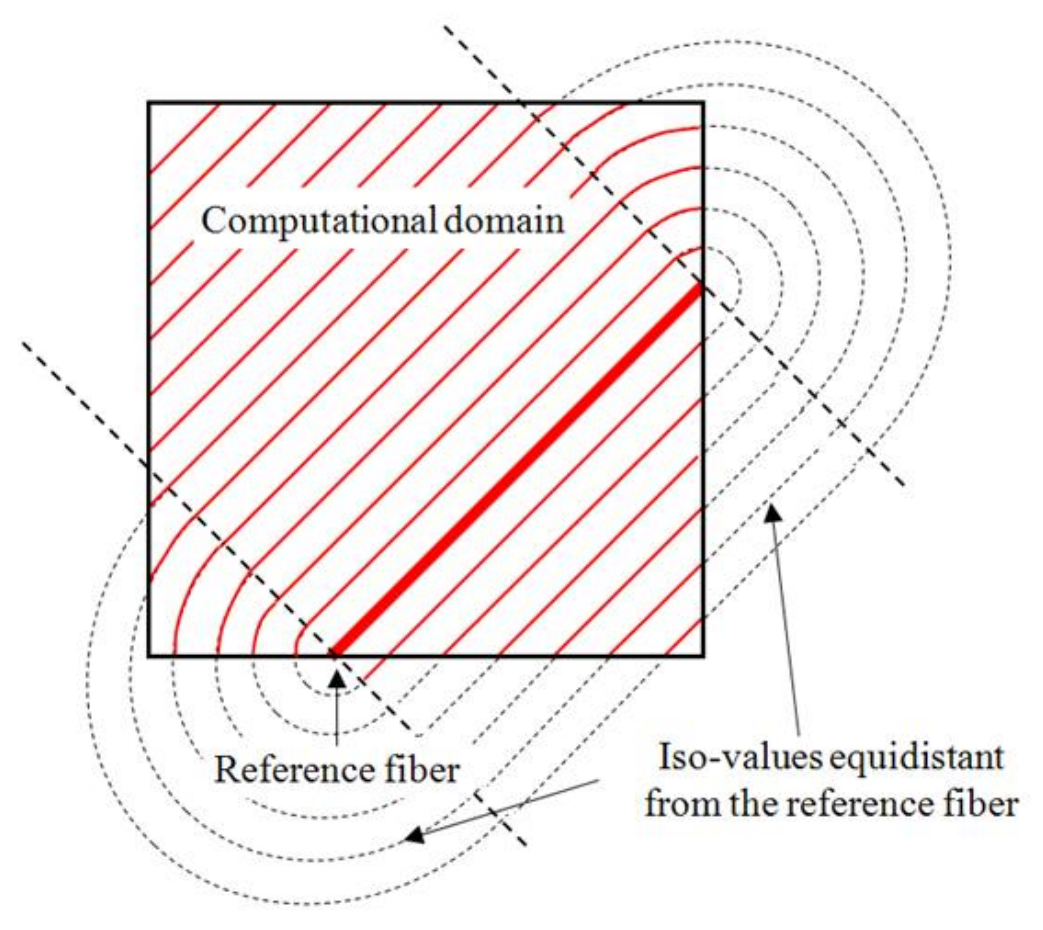

Figure 10: Propagation of a straight fiber segment on a square plate using the standard FMM: generation of undesired circles.

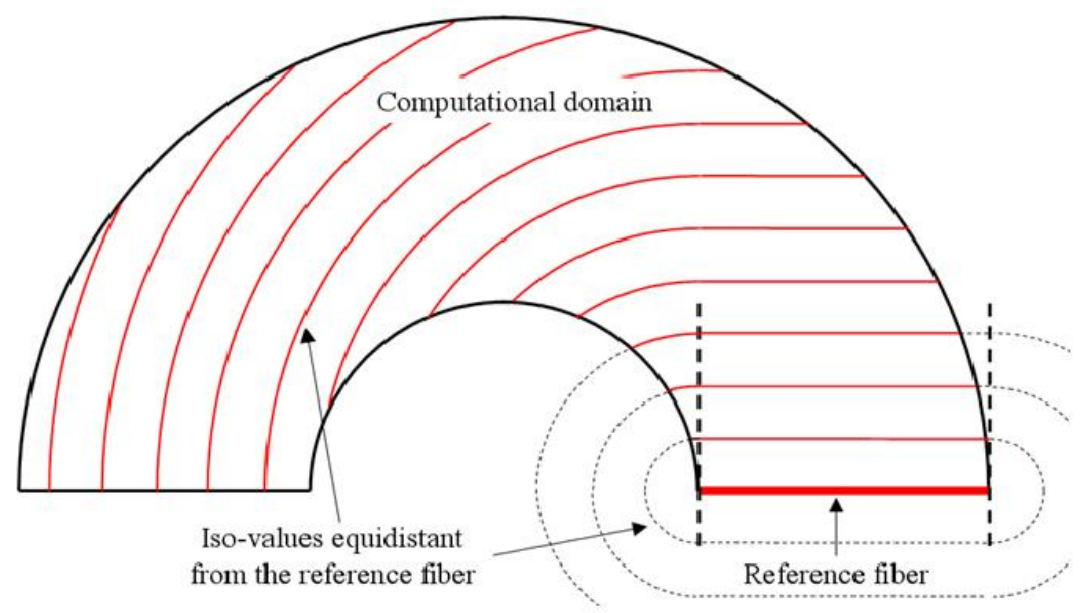

Figure 11: Propagation of a straight fiber segment on a cylinder (projected surface) using the standard FMM: generation of undesired circles. 


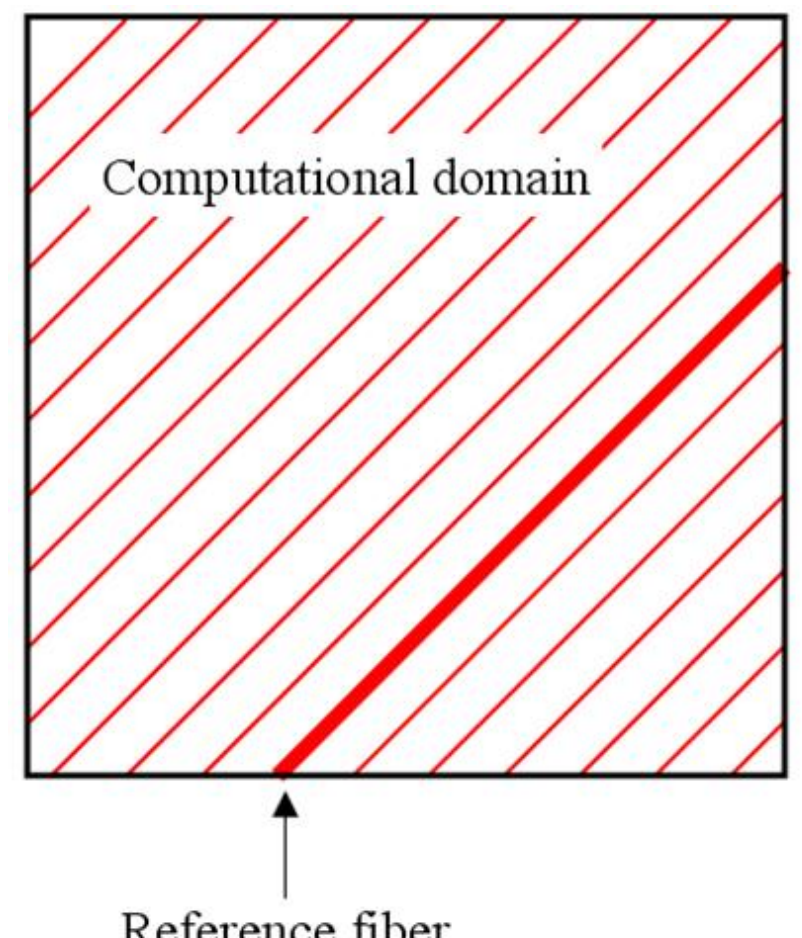

Figure 12: Expected network of fibers for the square plate.

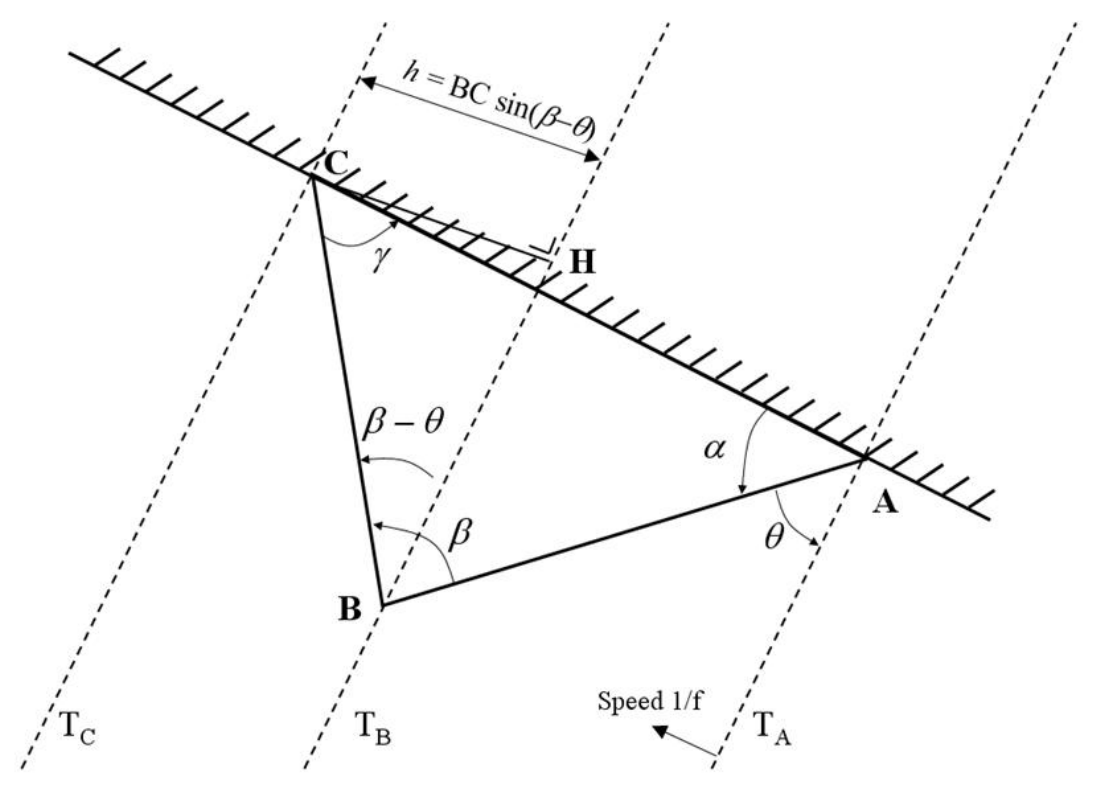

Figure 13: Adaptaiton of the FMM: updating $C$ from outside the domain in the case of an infinite fiber. 


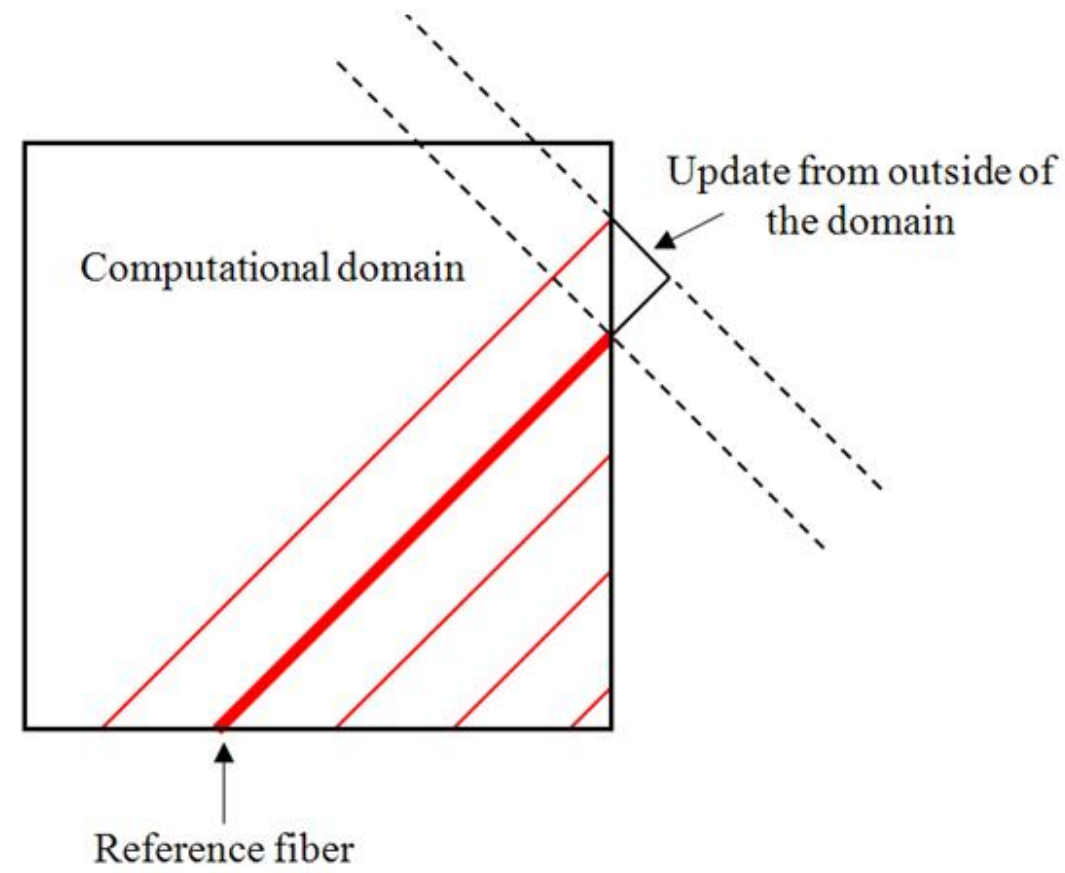

Figure 14: Propagation of a straight fiber on a cylinder (projected surface) with the adaptation described in Figure 13 .

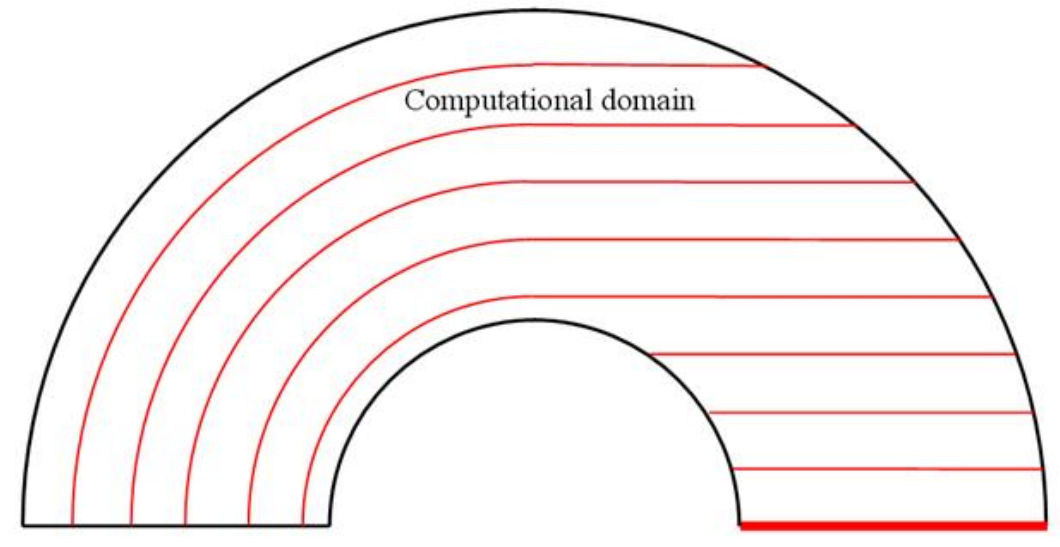

Figure 15: Propagation of a straight fiber segment using the modified FMM: simulation of an infinite reference fiber. 


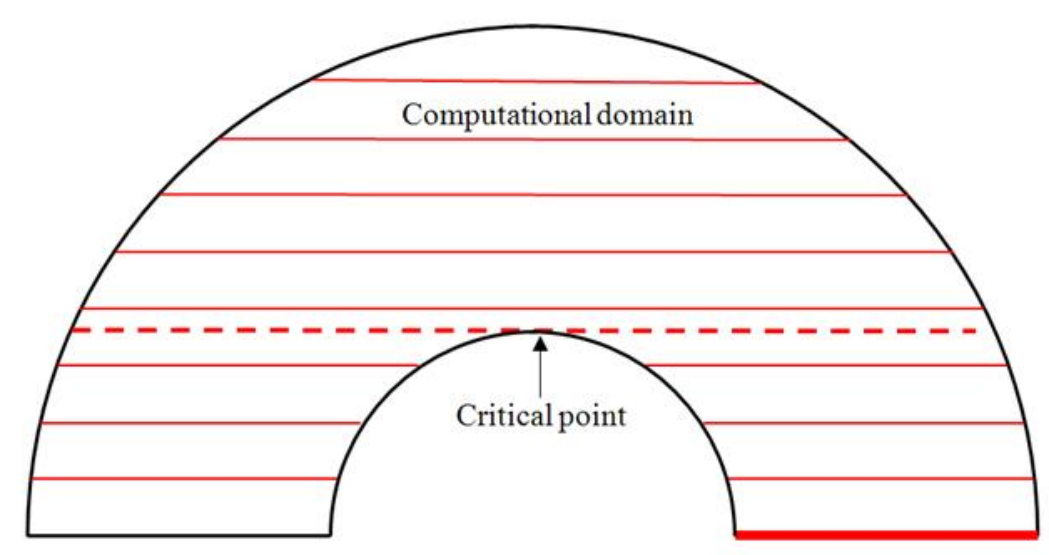

Figure 16: Case of a concave domain: extension of the fiber at the point of the boundary where the boundary is parallel to the fiber.

$T_{M}, T_{N}$ and $T_{P}$ at $A, B$ and $C$. The fiber inside the triangle is orthogonal to the gradient of $\tilde{T}$, thus, its orientation is the vector $u=(-b, a)$. The angle $\theta$ between the fiber and $A B$ is given by

$$
\cos \theta=\frac{\overrightarrow{A B} \cdot \vec{u}}{\|A B\| \cdot\|u\|}
$$

Let $\vec{v}_{1}=\overrightarrow{A B} \times \overrightarrow{A C}$ and $\vec{v}_{2}=\overrightarrow{A B} \times \vec{u}$. The sign of theta is the sign of $\vec{v}_{1} \cdot \vec{v}_{2}$.

\section{Numerical Experiments}

In this section, we show the efficiency of our method to generate equidistant curved fibers on complex surfaces. It consists in tracing an reference fiber over the mesh of the surface and then using the modified fast marching method to solve the Eikonal equation. The algorithm is implemented in the pre-processor of the SAMCEF finite element code [19]. The computational time associated to the execution of the modified FMM is clearly negligible compared to a finite element analysis that may be conducted on the resulting composite model. The results figure presented here consist of figures showing the iso-values of $T(x)$ 


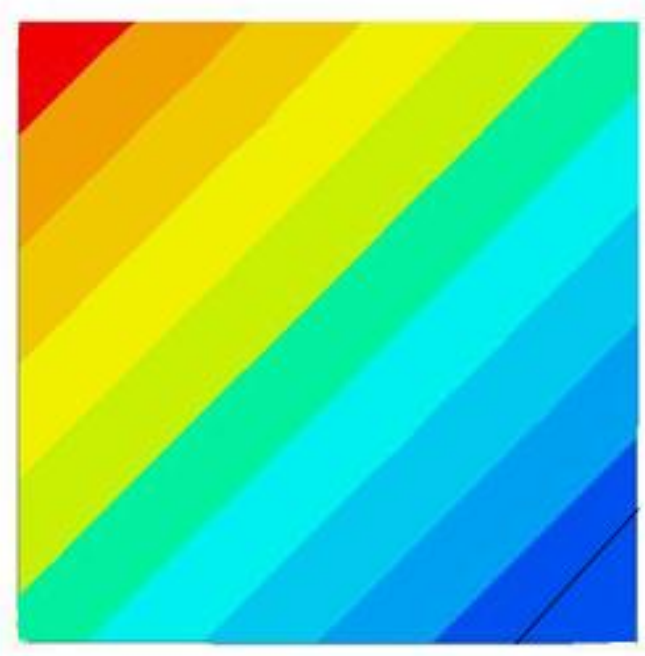

Figure 17: Straight reference fiber propagated over a square plate: illustration of the first adaptation of the FMM.

over the surface which represent the equidistant courses of the fiber placement machine. It is important to note that each colored band in the figures is a course of the AFP machine.

As a first example, the problem of Figure 12 is solved. The reference curve is a straight line, which is propagated over a rectangular domain, as shown in Figure 17. This example illustrates the first adaptation of the FMM by simulating the propagation of an infinite straight line rather than a bounded segment like with the standard FMM. Figure 17 shows the propagation of the straight line without the generation of circles as the standard FMM would have done in Figure 10.

The second example shows the propagation of an infinite straight fiber over a cone (Figure 18). This case requires the second adaptation of the FMM which is the prolongation of the fiber at some critical point of the boundary of the cone. It is the same case here as the one in figure 16. The light green fiber is tangent to the boundary of the cone. This particular fiber is prolongated at tangency point. Therefore, all the fibers from yellow to red are computed based 


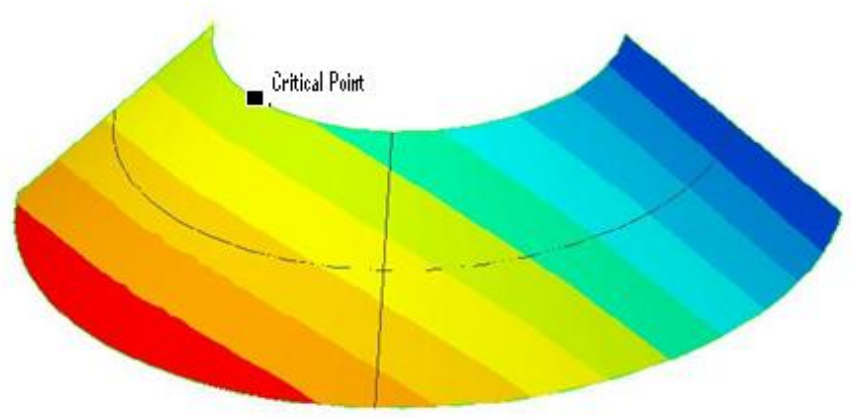

Figure 18: Straight reference fiber propagated over a cone: illustration of the second adaptation of the FMM.

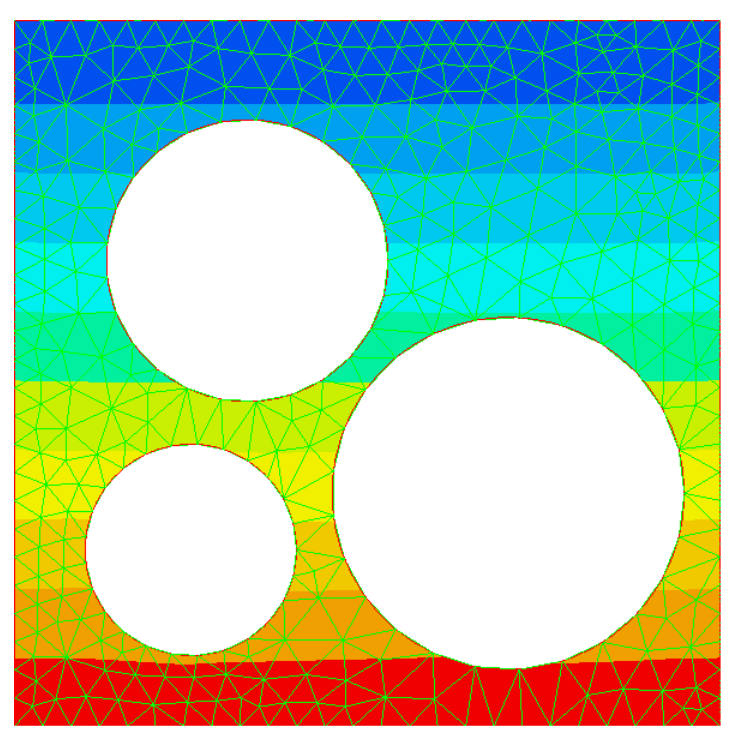

Figure 19: Straight fiber over a rectangle with holes.

on this prolongation. As a result, all the domain is filled with equidistant straight fibers.

The third example shows the benefits of using a mesh for describing the structure rather than geometric equations (Figure 19). Here we have a complex geometry which is a rectangle with three holes and straight equidistant fibers parallel to one edge. The algorithm uses the mesh of this surface and not its geometric definition. Thus, the user does not have to specify the three holes or any other particularities of the geometry as with the methods based on the geometry, since all the information is contained in the mesh. 

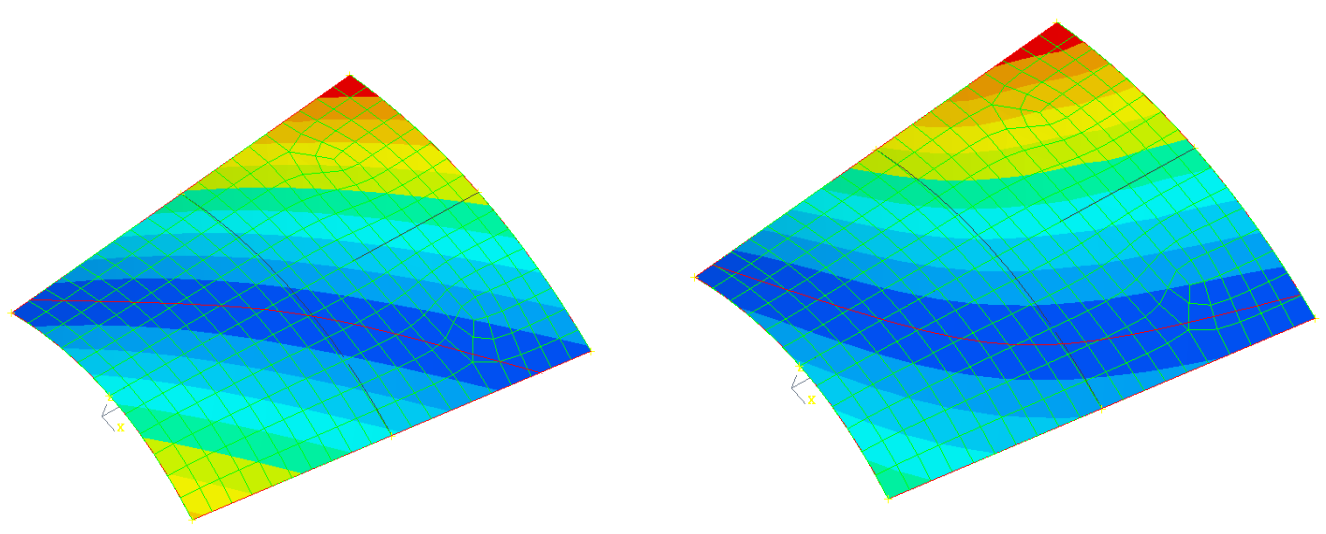

Figure 20: Straight fiber over a non- Figure 21: Curved fiber over a non-developable developable surface. surface.

Figures 20 to 23 are examples of straight and curved fibers over complex and non-developable surfaces. The first two figures show the mesh that is used to solve the Eikonal equation. Note that the quadrangular elements are divided into triangles in order to run the FMM. Again, these figures show the efficiency of the developed method to generate equidistant fibers over general surfaces coming from industrial cases.

Finally, Figure 24 shows the computation of the orientation of the fiber in each mesh element in the case of a non developable surface as described in Section 7. This provides the input for a finite element analysis of the composite structure.

\section{Conclusion}

In this paper, an algorithm is presented for determining equidistant (and possibly curved) fiber trajectories over 3D surfaces, avoiding gaps and overlaps in the definition of the plies but laying down courses of constant (and maximum) width. It is based on a modified version of the Fast Marching Method (FMM), which is a numerical method used for the simulation of 

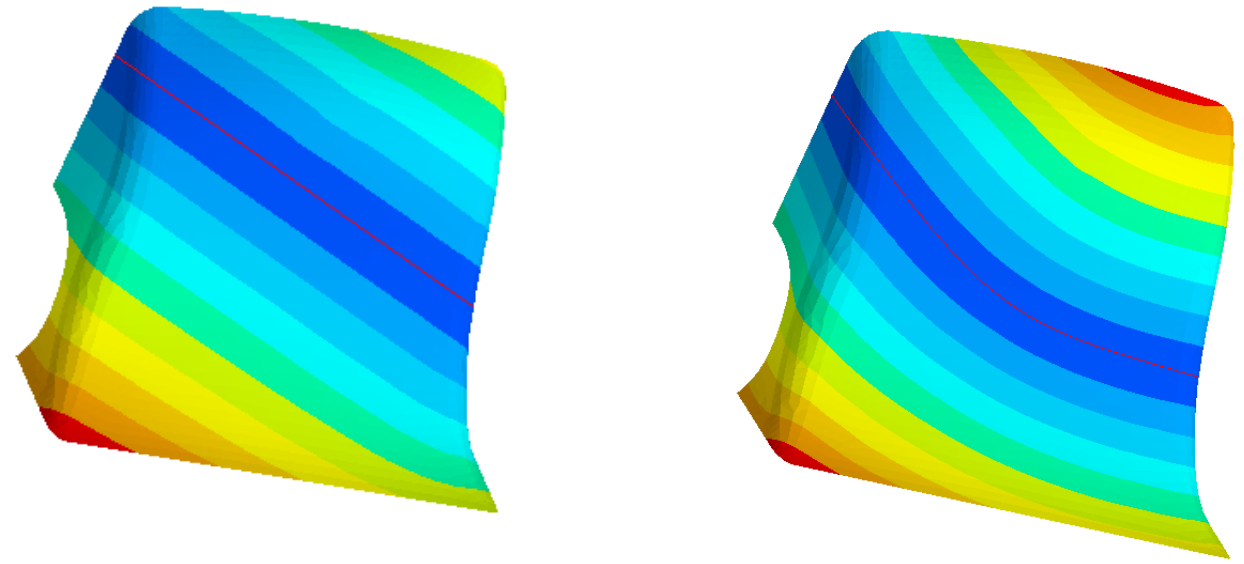

Figure 22: Straight fiber over a complex ge- Figure 23: Curved fiber over a complex geomometry. etry

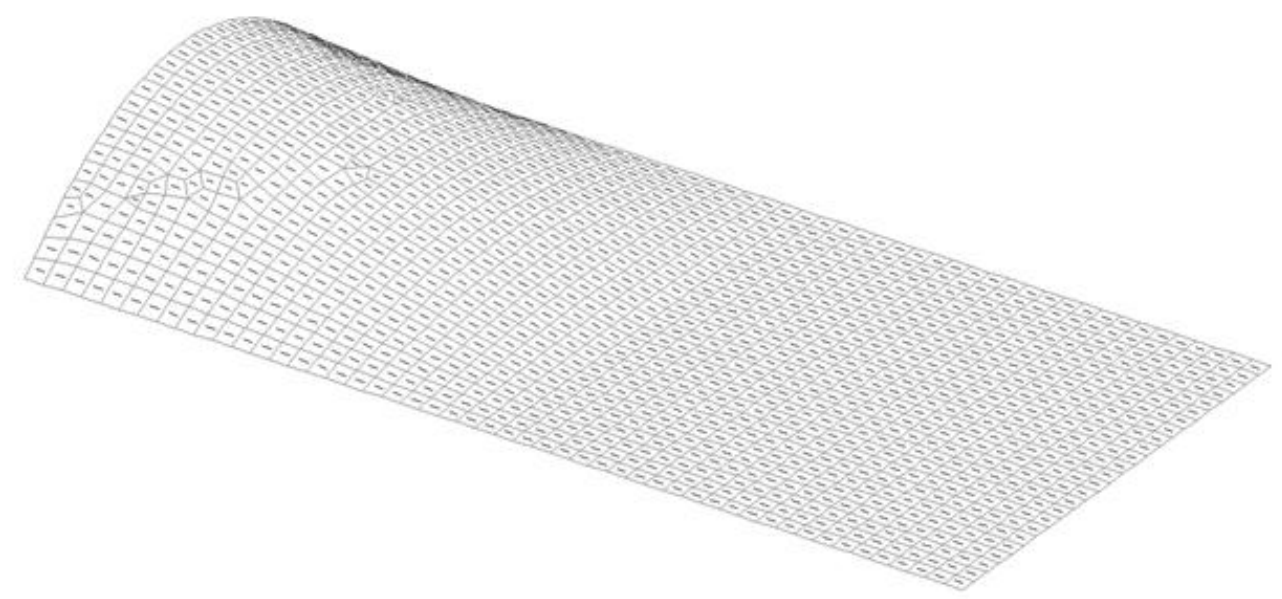

Figure 24: Resulting fiber orientations of each mesh element for a finite elements analysis. 
moving interfaces. Adaptations of the standard FMM have been proposed, so that the developed algorithm can provide suitable trajectories for an AFP machine. The algorithm has the advantage of working on a mesh for describing the surface rather than on its parametric equation. Moreover, it computes the fiber orientation in each element of the mesh, what is relevant for a finite elements analysis. The numerical experiments have demonstrated the efficiency of this approach to generate equidistant fiber trajectories without gaps and overlaps, in the cases of straight and curved fibers defined over 3D surfaces of complex geometries coming from industrial cases.

\section{Acknowledgments}

The authors would like to thank the Walloon region of Belgium and Skywin (Aerospace Cluster of Wallonia) for their support through the project VIRTUALCOMP.

\section{References}

[1] Grant C., "Automated processes for composite aircraft structure", Industrial Robot, 33(2), pp. 117-121 (2006).

[2] Blom A.W., Stickler P;B., and Gürdal Z., "Optimization of a composite cylinder under bending by tailoring stiffness properties in circumferential direction", Composites: Part B, 41, pp. 157-16 (2010).

[3] Van West B.P., Pipes R.B., Keefe M., "A simulation of the draping of bidirectional fabrics over arbitrary surfaces", Journal of the textile Institute, 81(4), pp. 448-460 (1990).

[4] Wang J., Paton R., Page J.R., "The draping of woven fabric performs for 
production of polymer composite components", Composites: Part A, 30, pp. 757-765 (1999).

[5] Dong L., Lekakou C., Bader M.G., "Solid-mechanics finite element simulations of the draping of fabrics: a sensitivity analysis",Composites: Part A, 31, pp. 639-652 (2000).

[6] Potter K. "Beyond the pin-jointed net: maximizing the deformability of aligned continuous fiber reinforcements",Composite Part A, 33, pp. 677-686 (2002).

[7] Tatting B., and Gürdal Z., "Design and manufacture of elastically tailored tow placed plates", NASA/CR-2002-211919 (2002).

[8] Hyer M., Charette R., "The use of curvilinear fiber format in composite structures design", Proceedings of the 30th AIAA/ASME/ASCE/AHS/ASC Structures, Structural Dynamics, and materials (SDM) Conference, New York, NY, paper 1404 (1989).

[9] Gürdal Z., Olmedo R., "In-plane response of laminates with spatially varying fiber orientations: variable stiffness concept", AIAA Journal, 31(4), pp. 751-758 (1993).

[10] Tatting, B. F., Gürdal, Z., "Design and Manufacture of Tow-placed Variable Stiffness Composite Laminates with Manufacturing Considerations", Proceedings of the 13th U.S. National Congress of Applied Mechanics (USNCAM), Gainesville, FL, June 21-26 (1998).

[11] Parnas L., Oral S., Ceyhan U., "Optimum design of composite structures with curved fiber courses", Composites Science and technology, 63, pp. 1071-1082 (2003). 
[12] Blom A.W., Tatting B.F., Hol J.M.A.M., Gürdal Z., "Fiber path definitions for elastically tailored conical shells", Composites: Part B, 40, pp. 77-84 (2009).

[13] Shirinzadeh B., Cassidy G., Oetomo D., Alici G., and Ang M.H., "Trajectory generation for open-contoured structures in robotic fiber placement", Robotics and Computer-Integrated Manufacturing, 23, pp. 380-394 (2007).

[14] Waldhart, C. J., Gürdal, Z., Ribbens, C., "Analysis of Tow-placed, Parallel Fiber, Variable Stiffness Laminates", Proceedings of the 37th AIAA/ASME/ASCE/AHS/ASC Structures, Structural Dynamics, and Materials (SDM) Conference, Salt Lake City, UT, April 15-17, pp. 2210-2220 (1996).

[15] Waldhart, "Analysis of tow-placed, variable-stiffness laminates", Master Thesis, Virginia Polytechnic Institute and State University, Blacksburg, USA (1996).

[16] Sethian J.A., "Level-set methods and fast marching methods: evolving interfaces in computational fluid mechanics, computer vision, and materials science", Cambridge University Press (1999).

[17] Zienkiewicz OC., "The finite element method", 3rd edn. McGraw-Hill, New York. (1977).

[18] Qian J., Hong Y.T., Zhao K., "Fast Sweeping Methods for Eikonal Equations on Triangular Meshes", SIAM J. Numerical Analysis 45(1): 83-107 (2007).

[19] SAMCEF. www.samtech.com 


\section{List of Figures}

1 Gaps and overlaps between two consecutive fiber courses due to non-equidistant fibers. . . . . . . . . . . . . 5

2 Propagation of the initial curve and equidistant curves at different instants. ..................... 6

3 Define the mesh of the structure. . . . . . . . . . . . 7

4 Trace the reference fiber. . . . . . . . . . . . . . 7

5 Solve the Eikonal equation. . . . . . . . . . . . . . 7

6 Compute the fiber orientation for each element. . . . . . . . . . 7

$7 \quad$ Tracing the reference fiber over the mesh from $x(t) \ldots \ldots$. . . . 8

$8 \quad$ Updating $T_{C}$ from $T_{A}$ and $T_{B} \ldots \ldots \ldots \ldots$

9 The fast marching method: the froze nodes (black dots), the close nodes (white squares) and the far nodes (white dots). . . . . . . 12

10 Propagation of a straight fiber segment on a square plate using the standard FMM: generation of undesired circles. . . . . . . . . 14

11 Propagation of a straight fiber segment on a cylinder (projected surface) using the standard FMM: generation of undesired circles. 14

12 Expected network of fibers for the square plate. . . . . . . . . . 15

13 Adaptaiton of the FMM: updating $C$ from outside the domain in the case of an infinite fiber. . . . . . . . . . . . . 15

14 Propagation of a straight fiber on a cylinder (projected surface) with the adaptation described in Figure 13 . . . . . . . . . . 16

15 Propagation of a straight fiber segment using the modified FMM: simulation of an infinite reference fiber. . . . . . . . . . . 16 
16 Case of a concave domain: extension of the fiber at the point of the boundary where the boundary is parallel to the fiber. . . . . . 17

17 Straight reference fiber propagated over a square plate: illustration of the first adaptation of the FMM. . . . . . . . . . . . 18

18 Straight reference fiber propagated over a cone: illustration of the second adaptation of the FMM. . . . . . . . . . . . . . . . 19

19 Straight fiber over a rectangle with holes. . . . . . . . . . . . . . . 19

20 Straight fiber over a non-developable surface. . . . . . . . . . . . . 20

21 Curved fiber over a non-developable surface. . . . . . . . . . . . . 20

22 Straight fiber over a complex geometry. . . . . . . . . . . . . . . . 21

23 Curved fiber over a complex geometry . . . . . . . . . . . . . 21

24 Resulting fiber orientations of each mesh element for a finite elements analysis. .................... . . . . 21 